\title{
Effect of biochar amendment on soil's retention capacity for estrogenic hormones from poultry manure treatment
}

\author{
Sukhjot MANN ${ }^{1}$, Zhiming QI (凶) ${ }^{1}$, Shiv O. PRASHER ${ }^{1}$, Lanhai LI $^{2}$, Dongwei GUI ${ }^{2}$, Qianjing JIANG ${ }^{1}$ \\ 1 Department of Bioresource Engineering, McGill University, Quebec H9X 3V9, Canada \\ 2 Xinjiang Institute of Geography and Ecology, Chinese Academy of Sciences, Urumqi 830001, China
}

\begin{abstract}
Most animals, including humans, produce natural sex hormones such as estrogens: $17 \beta$-estradiol (E2) and estrone (E1). These compounds are able to disrupt the reproductive systems of living organisms at trace concentrations $\left(\mathrm{ng} \cdot \mathrm{L}^{-1}\right)$. This experiment tests the hypothesis that $1 \%$ slow pyrolysis biochar-amended sandy soil could retain significant amount of estrogens (E1, E2) from poultry manure in its second year of application. The experiment was conducted over 46 days and consisted of a series of lysimeters containing sandy soil with biocharamended topsoil. The application rate of poultry manure was kept at $2.47 \mathrm{~kg} \cdot \mathrm{m}^{-2}$. The biochar held a significant concentration of hormone during the first year of its application. However, in the following year (current study), there was no significant retention of hormones in the biochar-amended soil. During the first year after application, the biochar was fresh, so its pores were available for hydrophobic interactions and held significant concentration of hormones. As time passed there were several biotic and abiotic changes on the surface of the biochar so that after some physical fragmentation, pores on the surface were no longer available for hydrophobic interactions. The biochar started releasing dissolved organic carbon, which facilitated greater mobility of hormones from poultry manure down the soil profile.
\end{abstract}

Keywords adsorption, degradation, dissolved organic carbon, 17 $\beta$-estradiol (E2), estrone (E1)

\section{Introduction}

Over the last few decades, an excessive quantity of organic

Received October 3, 2016; accepted January 24, 2017

Correspondence: zhiming.qi@megill.ca wastes (animal manure and biosolids) has been generated due to an intensification of anthropogenic activities and other modern animal feeding operations. Animal manures are commonly applied to agricultural fields as fertilizers. Though there are benefits of using organic waste for agricultural operations, the application of animal manure has been identified as one of the major gateways for the environmental exposure to new classes of toxic and highrisk organic contaminants known as emerging contaminants. Having endocrine-disrupting properties, some at concentrations as low as nanogram per liter, these contaminants represent a high chronic toxicity risk and are associated with adverse long-term health issues, such as carcinogenicity, mutagenicity and teratogenicity in aquatic animals especially fish ${ }^{[1-3]}$. Aquatic species like trout, turtles and minnow, may be sexually disabled when exposed to estrogens even at trace concentrations of nanogram per $\operatorname{liter}^{[4]}$. Natural steroidal sex hormones, estrogens including $17 \beta$-estradiol (E2) and estrone (E1), are the emerging contaminants of the greatest concern due to their adverse developmental and carcinogenic effects. A major source of estrogens in the environment comes from animal manure applied as fertilizer ${ }^{[5]}$. There is a wide recognition of the contamination threat caused by nutrient leaching from manure-amended agricultural fields but little is known about the leaching of estrogens through the soil to either shallow groundwater or surface water (the latter via drainage water). Due to their endocrine disrupting properties, the estrogens, E2 and its metabolite estrone, E1, has become a potential environmental concern. On average, rainfall is more frequent during the spring season. The application rate of manure during this season is also frequent meaning that the probability of leaching of estrogens to ground water is high during this period of time. Recent environmental studies have therefore focused on the prevalence, fate, transport pathways and ecotoxicology of these manure-borne hormones.

Various studies have found sorbents that demonstrated 
the removal of contaminants that are toxic for the environment ${ }^{[6,7]}$. Some of these studies have shown significant adsorption potential of biochar for hormones ${ }^{[8]}$. Biochar has carbon residues resulting from the pyrolysis of biomass $^{[9]}$. The $60 \%-80 \%$ black carbon in biochar and its high surface area ${ }^{[10]}$ allows this engineered sorbent to be potentially used as a remediation amendment to curb hormonal pollution in and from agricultural fields. In several studies, biochar has been shown to have adsorbing potential for hormones, but the duration for which this works at the same capacity is unknown. Tests on the capacity of biochar to retain hormones from poultry manure were done over two consecutive years. Studies on the adsorption potential of fresh $1 \%$ slow pyrolysis biochar for hormones from poultry manure in our specific field parameters were previously reported ${ }^{[11]}$. Therefore, the objective of this study was to investigate the transport of estrogens from poultry manure in $1 \%$ slow pyrolysis biochar-amended sandy soil and explore the retention capacity of biochar for hormones during the second year after its application.

\section{Materials and methods}

\subsection{Study compounds}

Two types of female sex hormones (estrogens) were observed in the study, 17 $\beta$-estradiol, commonly known as
E2 and its primary metabolite, estrone commonly known as E1. The analytical chemical standards for E2 ( $\geqslant 98 \%$ purity) and E1 ( $\geqslant 99 \%$ purity) were purchased from Sigma Aldrich (St. Louis, MO, USA). Double-deionised water (Milli-Q Millipore, Molsheim, France) was used in the preparation of standard solutions and mobile phase solutions. HPLC (high performance liquid chromatography) grade acetonitrile, which was used as a solvent in both the extraction process and for the mobile phase in HPLC induced separation process, was purchased from Fisher Scientific (Fair Lawn, NJ, USA). The physical and chemical properties, and molecular structures of E2 and E1 are shown in Table 1.

\subsection{Soil Characteristics}

The experiment was conducted at the Macdonald Farm of McGill University, Sainte Anne De Bellevue, Quebec, Canada using the sandy soil of the Saint-Amable complex found on site. The soil was packed into the lysimeters with the dimensions shown in Fig. 1. The lysimeters were kept outdoors under natural conditions but under a canopy to achieve controlled irrigation. Table 2 shows the physical and chemical properties of soil used for experiment.

This study is independent of any plant absorbing parameters. The reason for not using any plant is that our prime focus was to analyze only the retaining capacity of biochar. Growing plants would have changed the behavior of hormones in the soil.

Table 1 Physiochemical properties of estrogens ${ }^{[12]}$

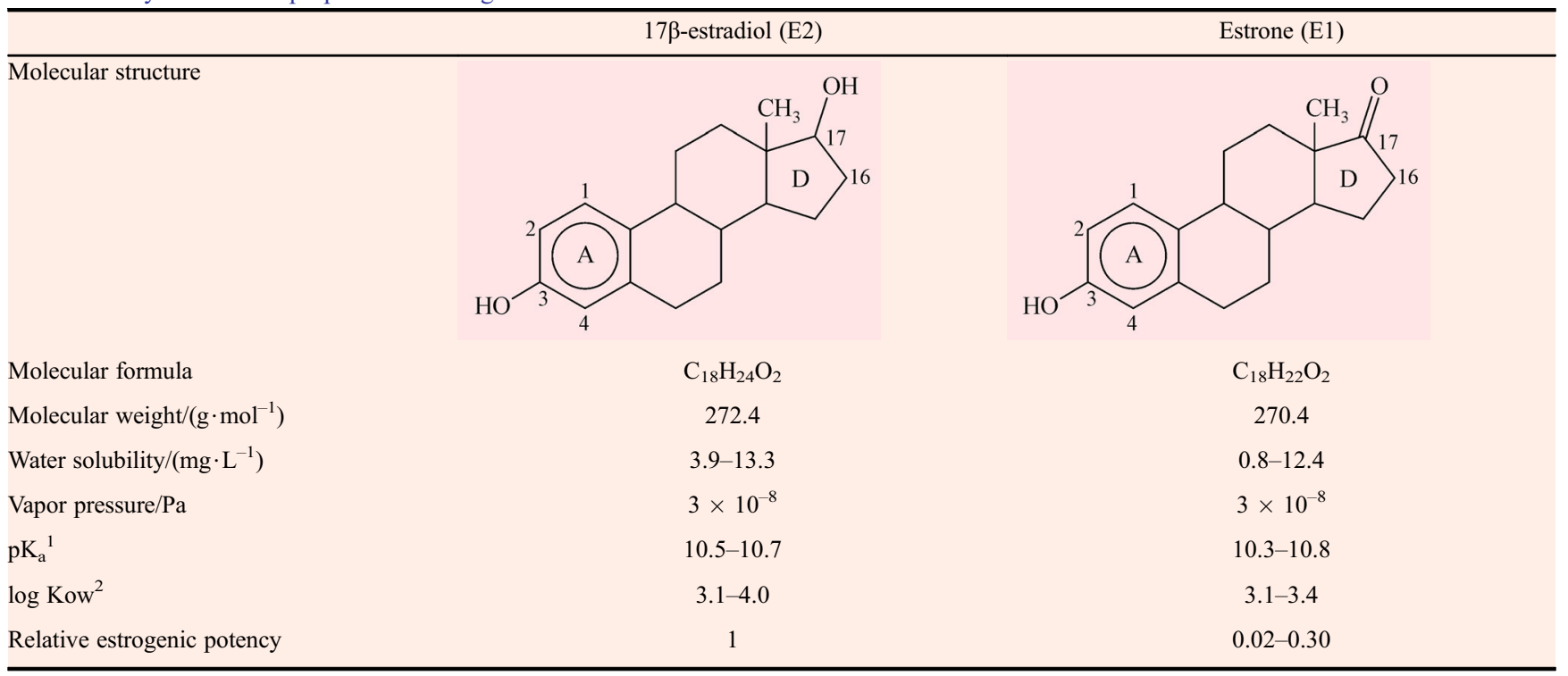

Note: ${ }^{1}$ Acid ionization constant; ${ }^{2}$ octanol-water partition coefficient.

Table 2 Physical and chemical properties of soil

\begin{tabular}{lccccccc}
\hline Soil type & Sand $/ \%$ & Silt $/ \%$ & $\mathrm{pH}$ & Bulk Density $/\left(\mathrm{kg} \cdot \mathrm{m}^{-3}\right)$ & $\mathrm{OM}^{\mathrm{a}} / \%$ & $\mathrm{CEC}^{\mathrm{b}} /\left(\mathrm{cmol} \cdot \mathrm{kg}^{-1}\right)$ & $\mathrm{HC}^{\mathrm{c}} /\left(\mathrm{cm} \cdot \mathrm{d}^{-1}\right)$ \\
\hline Sand & 92.2 & 4.3 & 5.5 & 1350 & 2.97 & 4.9 \\
\hline
\end{tabular}

Note: ${ }^{\text {a }}$ Organic matter; ${ }^{\mathrm{b}}$ cation exchange capacity; ${ }^{\mathrm{c}}$ hydraulic conductivity with Standard Deviation of 0.45 . 


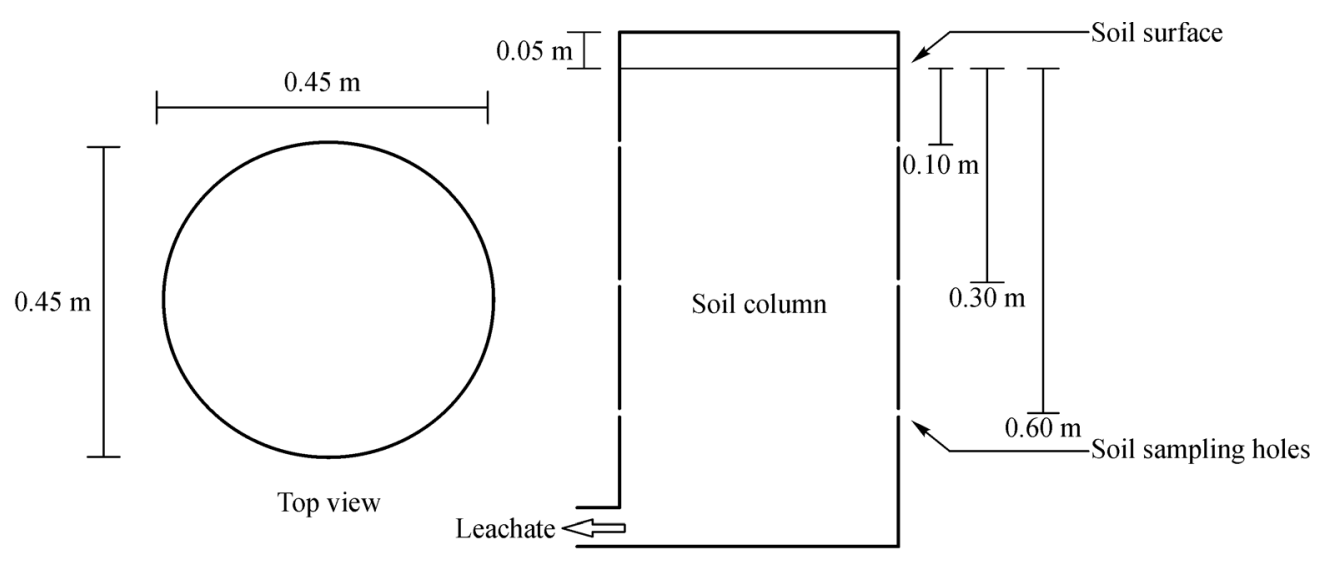

Side view

Fig. 1 Schematic design of the lysimeter

\subsection{Biochar}

The biochar used in this study was produced by the slow pyrolysis of soft wood at $450^{\circ} \mathrm{C}$ and bought from BlueLeaf Inc., Drummondville, Quebec, Canada. The biochar was applied as an Esoil amendment in the lysimeters in the summer of 2012. One percent $(\mathrm{w} / \mathrm{w})$ of biochar was mixed with topsoil with an application rate of $10 \mathrm{t} \cdot \mathrm{hm}^{-2[8]}$. Some properties of this biochar as determined by Control Laboratories Inc., Watsonville, California, USA, are given in Table 3.

\subsection{Experimental setup}

The field experiment investigating hormone transport with biochar present in a sandy soil was conducted with six outdoor PVC lysimeters. The experiment was conducted between June and August 2013. The lysimeters $(0.45 \mathrm{~m}$ I.D. $\times 1.00 \mathrm{~m}$ high) were sealed at the bottom by $0.6 \mathrm{~m} \times$ $0.6 \mathrm{~m}$ PVC sheets. Each lysimeter was packed in layers of sand and adjusted to a bulk density of $1350 \mathrm{~kg} \cdot \mathrm{m}^{-3}$. A drainage pipe ( $50 \mathrm{~mm}$ diameter) was installed at the bottom of each lysimeter. To take composite sample from each depth, four horizontal soil sampling holes were made in each lysimeter at depths of $0.10,0.30$ and $0.60 \mathrm{~m}$ from the surface of the soil (Fig. 1). To prevent any plant uptake, plant residues were removed from the soil surface and no crops were planted. A canopy was installed over the lysimeters to protect them from rain and protect from storms. Two treatments were applied, (1) soil only (control) without any added biochar and (2) topsoil amended with biochar. Three replicate lysimeters were established for each treatment.

\subsubsection{Manure application}

Poultry manure was obtained from the Burnbrae Farms situated at 200 69th Avenue St-Zotique, Quebec. The application rate of the poultry manure used in this study was calculated based on the recommendations of Beegle $^{[13]}$, Pennsylvania State University, USA. The main factors in manure application rate estimation include crop nitrogen requirement, nitrogen availability of the manure, and application time. The application rate of the poultry manure was calculated for silage corn with the typical yield of $4.94 \mathrm{~kg} \cdot \mathrm{m}^{-2}$ and nitrogen requirement for given yield is $0.02 \mathrm{~kg} \cdot \mathrm{m}^{-2}$. Taking spring as the application time, incorporation into the topsoil as the application method, average total nitrogen content of poultry manure to be $0.0168 \mathrm{~kg} \cdot \mathrm{kg}^{-1}$ and 0.50 as the nitrogen availability factor when incorporation of manure was done within 1 day, the estimated application rate of poultry manure was found to be $2.47 \mathrm{~kg} \cdot \mathrm{m}^{-2}$. After conversion to the lysimeter area, approximately $0.392 \mathrm{~kg}$ of poultry manure was added to each lysimeter.

\subsubsection{Soil and leachate sampling}

Before starting the experiment, all lysimeters were irrigated and brought to saturation. Soil samples were taken before applying manure to check any pre-existing hormones in the soil. Next day (Day 1), $0.392 \mathrm{~kg}$ of poultry

Table 3 Typical properties of the biochar sourced from BlueLeaf Inc, Drummondville, Quebec, Canada

\begin{tabular}{lccccc}
\hline $\mathrm{TA}^{\mathrm{a}}$ & Organic carbon & Inorganic carbon & Hydrogen/Carbon & Hydrogen $^{\mathrm{b}}$ & $\mathrm{TN}^{\mathrm{c}}$ \\
\hline $17.30 \%$ & $77 \%$ & $0.50 \%$ & 0.34 (molar ratio) & $2.20 \%$ & $0.56 \%$ \\
\hline
\end{tabular}

Note: ${ }^{\text {a }}$ Total ash; ${ }^{\mathrm{b}}$ the $\mathrm{H} / \mathrm{C}$ ratio indicates the degree of carbonization of biochar; ${ }^{\mathrm{c}}$ total nitrogen. 
manure was manually incorporated in the topsoil of each lysimeter. Soil sample was taken from the topsoil before the first irrigation to quantify hormones in topsoil after the addition of manure. A total of $9.67 \mathrm{~L}$ of tap water was applied at regular intervals over $4 \mathrm{~h}$ mimicking the rate of natural rainfall. All leachate that came from the drainage pipe was collected in amber colored bottles to avoid photodegradation of hormones in the sample. The next day (Day 2), composite soil samples were collected from the topsoil as well as at depths of $0.10,0.30$ and $0.60 \mathrm{~m}$ from the surface of soil. Soil samples were placed in airtight zip lock plastic bags. Soil samples were taken on six occasions; Day 0 (for background hormone concentration) and Days 1, 2, 16, 31 and 46 after application of the manure. The moisture of each soil sample was determined in a laboratory using thermal methods and the remaining portions of the samples were stored in sealed plastic bags in the freezer until extraction. After each of the four irrigation events on Days 1, 15, 30 and 45, approximately 7.5-8.0 L were collected at the bottom of each lysimeter. Leachate samples were then taken to the laboratory and the hormones were extracted from the leachate samples on the same day because the target hormones degrade faster in water than soil.

\subsubsection{Mass balance calculations}

For each sampling, the total mass of the hormones recovered was calculated by the equation of ElSayed et al. ${ }^{[14]}$, which states that the sum of the hormones recovered from soil across each depth of soil profile plus mass of hormones recovered from leachate sample would total the initial hormone mass present, minus any losses or unrecovered mass due to degradation or volatilization. The mass balance equation is:

$$
\begin{aligned}
H R M_{\text {Day } 1}= & A_{\text {cs }} \rho\left(C_{0.05-0.10} \theta_{0.05-0.10} h_{0.05-0.10}\right. \\
& +C_{0.10-0.20} \theta_{0.10-0.20} h_{0.10-0.20} \\
& +C_{0.20-0.50} \theta_{0.20-0.50} h_{0.20-0.50} \\
& \left.+C_{0.50-0.75} \theta_{0.50-0.75} h_{0.50-0.75}\right) \\
& +C_{\text {leach }} V_{\text {leach }} H R M_{\text {last }}
\end{aligned}
$$

where HRM is the mass of hormones ( $\mu \mathrm{g}), h_{x-y}$ is the depth of a given soil layer (m), calculated as $y-x, A_{\mathrm{cs}}$ is crosssectional area of the soil column, calculated as $\pi\left(\frac{D}{2}\right)^{2}$, where $D$ is the lysimeter inner diameter $(0.45 \mathrm{~m}), C_{\text {leach }}$ is the hormone concentration in the leachate $\left(\mu \mathrm{g} \cdot \mathrm{L}^{-1}\right), C_{x-y}$ is the hormone concentration $\left(\mu \mathrm{g} \cdot \mathrm{kg}^{-1}\right)$ in the soil layer ranging from $x$ to $y$ in depth (m), $V_{\text {leach }}$ is the volume of leachate (L), $\rho$ is the soil bulk density $\left(\mathrm{mg} \cdot \mathrm{m}^{-3}\right)$, and $\theta_{x-y}$ is the soil gravimetric moisture content in the soil layer ranging from $x$ to $y$ in depth (m).

\subsection{Hormone extraction methodology}

2.5.1 Method for hormones extraction from leachate samples

Hormones were extracted from leachate using solid phase extraction with Oasis HLB extraction cartridges (200 $\mathrm{mg} \cdot \mathrm{mL}^{-1}$, Oasis Co. Ltd, NY). A SPE vacuum manifold system was used to perform the extraction. To remove suspended particles, each 1-L subsample was filtered through a PreSep prefilter $47 \mathrm{~mm}$ (GE Water and Process Technologies, PA, USA). Prior to extraction, Oasis HLB extraction cartridges were conditioned with $5 \mathrm{~mL}$ of methanol and then followed by $5 \mathrm{~mL}$ of high purity water ${ }^{[15]}$. A flow rate of $20 \mathrm{~mL} \cdot \mathrm{min}^{-1}$ was maintained with the vacuum manifold while $1 \mathrm{~L}$ of sub sample was passed through a conditioned cartridge followed by a passage of $10 \mathrm{~mL}$ of HPLC grade acetonitrile. Along with the acetonitrile, hormones were eluted from cartridges. The extract was brought to dryness under a nitrogen stream and redissolved in $1 \mathrm{~mL}$ of 50:50 (V/V) water-acetonitrile solution. The glass tube containing the extract was put on a sonicator for $15 \mathrm{~min}$ to achieve homogeneous mixing. The extract was filtered through a sterile $0.22-\mu \mathrm{m}$ filter (MillexGV, Japan) using a syringe. The final extract was transferred into $1.5-\mathrm{mL}$ amber colored HPLC vials for HPLC analysis.

\subsubsection{Method for hormones extraction from soil samples}

Soil samples were stored frozen after collection from the field until hormone extraction. The soil samples were thawed at room temperature and the soil in each plastic zip lock bag was thoroughly mixed before extraction. The method for extraction of hormones from soil samples was adapted from that of Xuan et al. ${ }^{[16]}$. Five gram of each soil sample were taken and placed in $50 \mathrm{~mL}$ polyethylene centrifuge tubes. Ten mililiter of HPLC grade acetone and $5 \mathrm{~g}$ of anhydrous $\mathrm{Na}_{2} \mathrm{SO}_{4}$ were added to each tube containing the soil samples ${ }^{[16]}$. The materials were mixed thoroughly using a vortex mixer for $1 \mathrm{~min}$ followed by vigorous shaking for $30 \mathrm{~min}$ at $250 \mathrm{r} \cdot \mathrm{min}^{-1}$ in a reciprocating shaker. The mixture was centrifuged at $4000 \mathrm{r} \cdot \mathrm{min}^{-1}$ for $20 \mathrm{~min}$ in a centrifuge. After centrifugation, the supernatant was transferred to another clean $50 \mathrm{~mL}$ polyethylene centrifuge tube. Another $10 \mathrm{~mL}$ of acetone was added to the supernatant and the process was repeated. The supernatant obtained from the second round of centrifugation was transferred to another clean $50-\mathrm{mL}$ polyethylene centrifuge tube. The resultant supernatant was centrifuged again at $4000 \mathrm{r} \cdot \mathrm{min}^{-1}$ for $20 \mathrm{~min}$ and the final supernatant was transferred into $50-\mathrm{mL}$ pyrex glass centrifuge tubes and dried completely under a gentle $\mathrm{N}_{2}$ 
stream. The dried extract was redissolved in $1 \mathrm{~mL}$ of 50:50 $(\mathrm{V} / \mathrm{V})$ water-acetonitrile. The glass tube containing the extract was sonicated for 15 min to achieve homogeneous mixing. The extract was filtered through a sterile $0.22-\mu \mathrm{m}$ filters (Millex-GV, Japan). The final extract was transferred into $1.5-\mathrm{mL}$ amber colored HPLC vials for HPLC analysis.

\subsection{HPLC analysis}

The hormonal content of the leachate and soil samples was analyzed by using high pressure liquid chromatography (HPLC). Forty percent of Millipore water and $60 \%$ of HPLC grade acetonitrile was used as the mobile phase. A Zorbax Eclipse Plus C18 column $(150 \mathrm{~mm} \times 4.6 \mathrm{~mm})$ with particle size of $5 \mu \mathrm{m}$ from Agilent Technologies Inc (Santa Clara, CA, USA) was employed as the stationary phase. The gradient flow rate of the mobile phase was set at $1 \mathrm{~mL} \cdot \mathrm{min}^{-1}$. Run time for each injection was $15 \mathrm{~min}$. Each sample injection was $100 \mu \mathrm{L}$. The UV detector wavelength was $210 \mathrm{~nm}$.

\subsection{Data analysis}

The data was analyzed with PROC MIXED in SAS v. 9.2. This model used repeated measures to determine if hormone concentration differed between treatments over time and depth.

\subsection{Scanning electron microscopy (SEM)}

Scanning electron microscopy (SEM) was used to study the surface structural changes in biochar after two years in the soil. This analysis was carried using a Hitachi TM3000 Scanning Electron Microscope operating at $15 \mathrm{kV}$ bought from Olympus (Markham, ON, Canada). The images were taken at $250 \times$ magnification. SEM images are useful for obtaining accurate details about pore structure of biochars. Structural changes in biochar were analyzed by scanning the surface and inside fresh and used biochar samples. Scanning of the inside of the structure was achieved by gently dissecting the biochar before SEM examination.

\subsection{Sorption test}

A batch sorption experiment was performed to support the results obtained from the field experimentation. Sorption efficiencies for hormones were compared between fresh biochar and used biochar. Standard solutions of both $\mathrm{E} 1$ and $\mathrm{E} 2$ at six concentrations ranging between 0.01 to $5 \mathrm{mg} \cdot \mathrm{L}^{-1}$ were prepared in triplicate by adding calculated amounts of pure hormones (Sigma Aldrich, St. Louis, MO, USA) in HPLC grade acetonitrile. The method was adopted from a study conducted by Lee et al. ${ }^{[17]}$. Two grams of soil with $1 \%$ fresh slow pyrolysis biochar was equilibrated with $30 \mathrm{~mL}$ of each standard solution (of estrogen) in 50-mL glass centrifuge tubes with Teflonlined screw caps. The process was repeated with $1 \%$ used slow pyrolysis biochar recovered from field soil two years after application. Aluminum foil was wrapped around the tubes to avoid any photodegradation. All tubes were placed on a flat-bed shaker for $24 \mathrm{~h}$ in the dark at $23 \pm 2^{\circ} \mathrm{C}$.

\section{Results and discussion}

\subsection{Fate and transport of estradiol}

Mass balance calculations are shown in Table 4 . There was a sudden decrease in the mass of E2 after the first irrigation event in the control as well as the biochar treatment in the topsoil. However, it was clear that the rate of degradation in the topsoil decreased with time (Fig. 2a). This finding matches those of Casey et al. ${ }^{[18]}$, who reported that the rate of hormone degradation decreased with time and concentration.

Based on the mass balance table, the percentage of the mass of hormones held in the topsoil in biochar treatment and control for the current year of study was calculated and compared with the first year (Fig. 2a). During the first year, when biochar was freshly applied as a topsoil amendment, biochar held more mass of E2 in the topsoil (Fig. 2a) which allowed less mass of hormones from the poultry manure to leach down the soil profile into the lysimeter compared to the control. When the biochar was fresh, it had open pores

Table 4 Amount of estradiol E2 $(\mu \mathrm{g})$ at different soil depths ranges and in leachate, and overall, over $46 \mathrm{~d}$ period

\begin{tabular}{|c|c|c|c|c|c|c|c|c|c|c|c|c|}
\hline \multirow{3}{*}{ Day } & \multicolumn{12}{|c|}{ Treatment } \\
\hline & \multicolumn{6}{|c|}{ Soil + Poultry manure (Control) } & \multicolumn{6}{|c|}{ Soil + Poultry manure + Biochar } \\
\hline & $5-10 \mathrm{~cm}$ & $10-20 \mathrm{~cm}$ & $20-50 \mathrm{~cm}$ & $50-70 \mathrm{~cm}$ & Leachate & Total & $5-10 \mathrm{~cm}$ & $10-20 \mathrm{~cm}$ & $20-50 \mathrm{~cm}$ & $50-70 \mathrm{~cm}$ & Leachate & Total \\
\hline$\overline{0}$ & 3.88 & 0.00 & 71.86 & 61.08 & 0.000 & 136.82 & 6.12 & 12.2 & 54.1 & 25.7 & 0.000 & 98.13 \\
\hline 1 & 61.99 & & & & & & 128.2 & & & & & \\
\hline 2 & 43.26 & 7.84 & 17.93 & 54.08 & 0.760 & 123.86 & 50.85 & 9.33 & 75.95 & 0.00 & 0.223 & 136.35 \\
\hline 16 & 17.19 & 10.86 & 38.21 & 21.06 & 0.133 & 87.45 & 12.06 & 13.89 & 55.23 & 21.97 & 0.160 & 103.40 \\
\hline 31 & 6.19 & 34.35 & 22.40 & 12.42 & 0.240 & 75.61 & 7.98 & 5.31 & 27.11 & 35.16 & 0.269 & 75.82 \\
\hline 46 & 2.21 & 4.95 & 17.55 & 18.46 & 0.259 & 43.42 & 5.73 & 6.62 & 13.23 & 21.24 & 0.315 & 47.14 \\
\hline
\end{tabular}


(a)

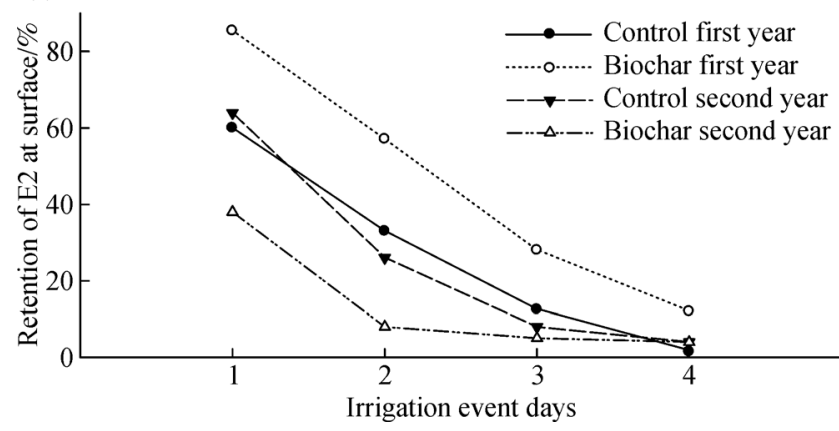

(b)

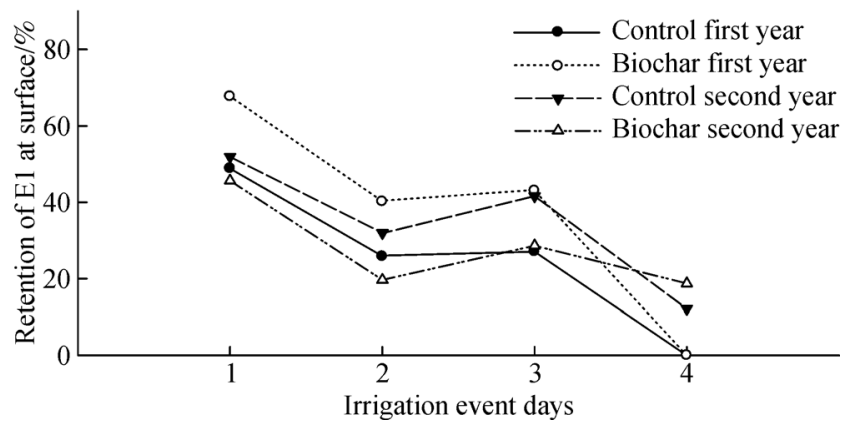

Fig. 2 Percentage of $17 \beta$-estradiol (E2) mass (a) and estrone (E1) mass (b) remaining in the topsoil (cumulative) during first year and second year

available for hydrophobic interactions which led to the adsorption of hormones onto the biochar particles. The relative hydrophobicity of estrogens, with $\log$ (octanolwater partition coefficient) values ranging from 2.6 to $4.0^{[19]}$ is a good indication of the strong sorption affinity of these hormones. Due to the hydrophobic properties of hormones ${ }^{[20]}$ they were held by the biochar in the topsoil. Unlike the first year, the biochar showed lower retention capacity for hormones in the second year after application and retained a reduced mass of E2 when compared to the control (Fig. 2a).

During the second year after biochar application, the control held comparatively more E2 in the topsoil when compared to the biochar treatment (Fig. 2a) during the first two irrigation events, although the degradation was nearly the same between both treatments (Fig. 3a) for this period. This indicates that a decrease in mass of E2 from the surface in biochar treatment is due to the leaching of E2 down the soil profile. Another reason for decreased mass of E2 over time is the transformation of E2 into E1 (estrone). Various studies ${ }^{[16]}$ suggested that E2 is metabolised into its daughter component E1.

There was more leaching in the biochar treatment than

(a)

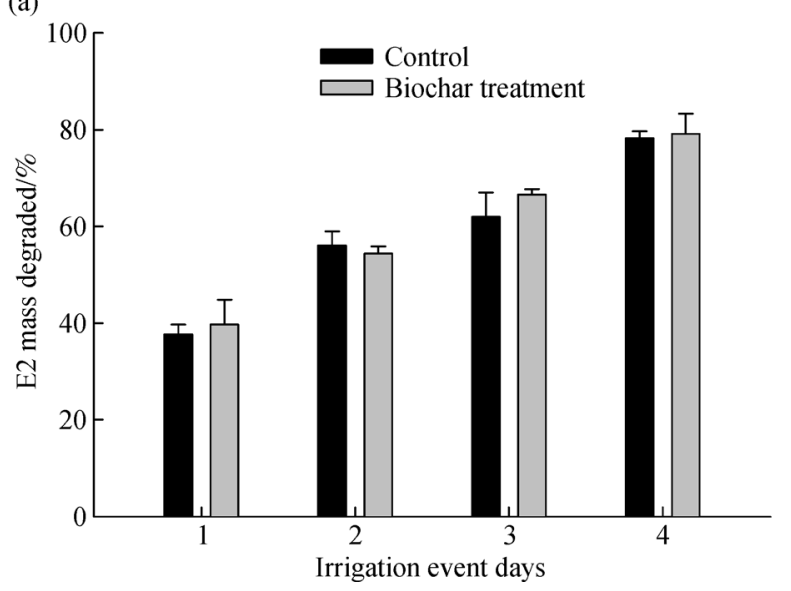

the control during the second year because of the release of dissolved organic carbon from the biochar. Given that the biochar was in the field for two years, there was some surface degradation as discussed below. Various studies have reported surface degradation of biochar over a period of time ${ }^{[21]}$. It was assumed in these studies that substances, such as humic acid, minerals, metal oxides and native pollutants, in the sediment had altered the physical or chemical properties of the biochar surface and affected the sorption of hydrophobic organic compounds. After initial fragmentation and undergoing various biotic and abiotic changes, the biochar released dissolved organic carbon, which has the potential to facilitate hormone movement down the soil profile ${ }^{[5]}$. This could explain why soil in the second year after biochar treatment showed increased mobility of the hormones down the soil profile compared to control.

3.1.1 Concentration of $17 \beta$-estradiol in soil at different depths and times

Figure 4 shows the concentration of $\mathrm{E} 2\left(\mu \mathrm{g} \cdot \mathrm{kg}^{-1}\right)$ in both

(b)

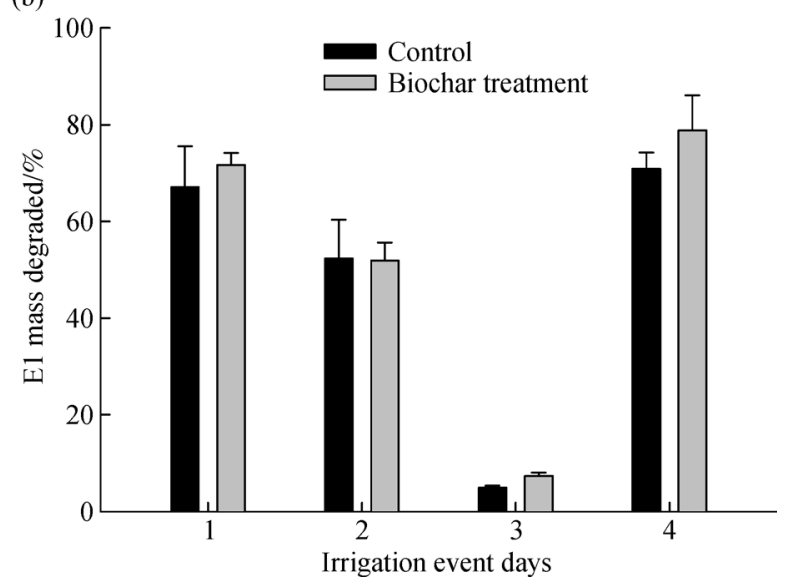

Fig. 3 17ß-estradiol (E2) mass (\%) (a) and estrone (E1) mass (\%) (b) degraded overall (cumulative) 

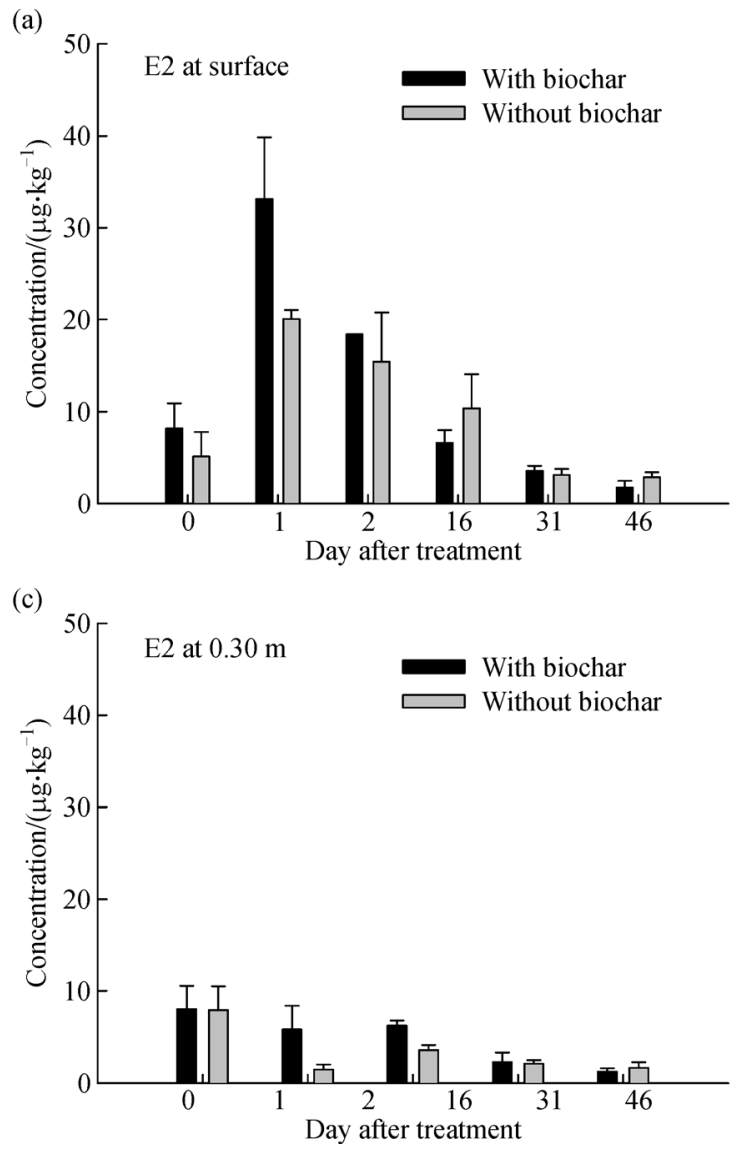
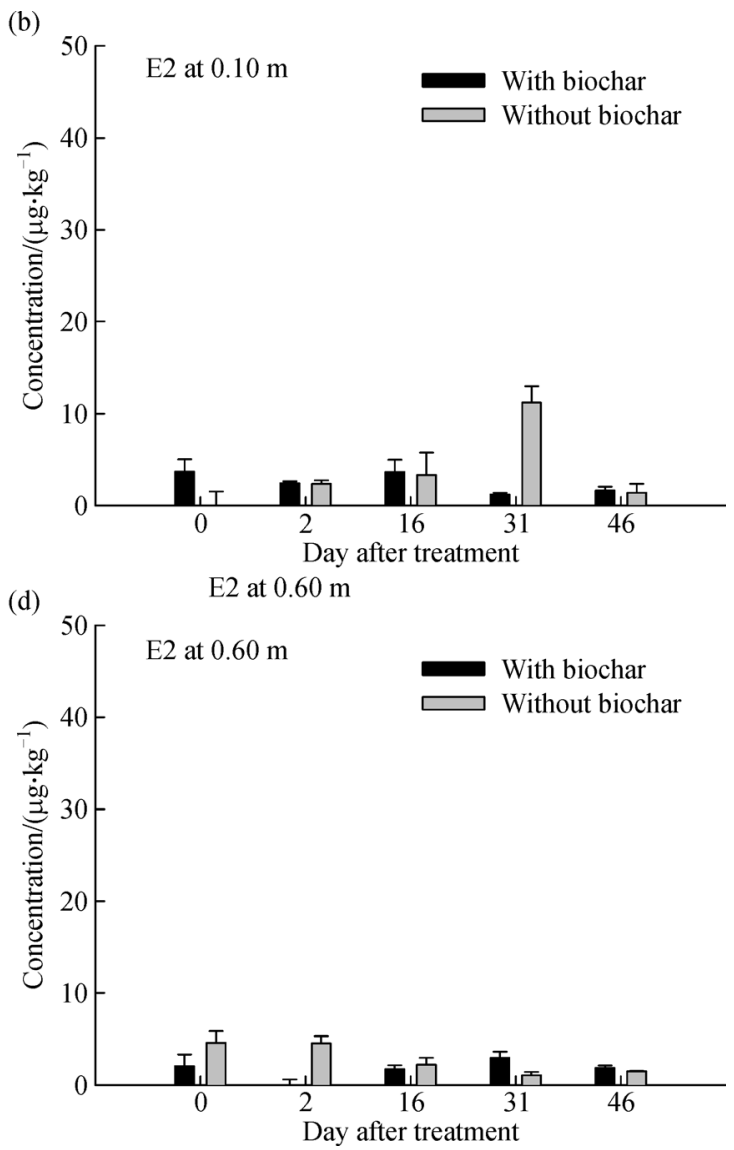

Fig. 4 17ß-estradiol $\left(\mu \mathrm{g} \cdot \mathrm{kg}^{-1}\right)$ concentration over a period of 46 days. (a) In the topsoil; (b) at $0.10 \mathrm{~m}$ from surface of soil profile; (c) at $0.30 \mathrm{~m}$; (d) at $0.60 \mathrm{~m}$.

treatments at different depths and times. The concentration of E2 was highest in the topsoil on Day 1 when the poultry manure was applied, then decreased over time in both treatments. In Fig. 4a, although the concentration of E2 in the biochar treatment on Days 1 and 2 was more than the control in the topsoil, the rate of E2 movement to lower depths during the following days was greater in the biochar treatment than the control. The rate of degradation of E2 decreased over time in both treatments, which matches the findings of Casey et al. ${ }^{[18]}$, who also found that the rate of hormone degradation decreased with time and concentration. The lower degradation rates of E2 in the biochar treatment at the last two irrigations was attributed to the presence of dissolved organic carbon which may provide an energy source $\mathrm{e}^{[22]}$.

In the presence of this energy source, microbial degradation of E2 was less significant. At other depths, there were no significant trends in concentration due to the decrease in mass of the hormones down the soil profile and the simultaneous increase in mass of hormones in the topsoil. For most days, the concentration of E2 at lower depths in both the control and the biochar treatments remained almost identical. These findings are supported by the statistical analysis shown in Table 5, which confirms
Table 5 Analysis of variance for $17 \beta$-estradiol and estrone residue in soil

\begin{tabular}{lcc}
\hline \multirow{2}{*}{ Effect } & \multicolumn{2}{c}{ Probability } \\
\cline { 2 - 3 } & $17 \beta$-estradiol (E2) & Estrone (E1) \\
\hline Treatment & $0.3396^{\mathrm{a}}$ & $0.6249^{\mathrm{a}}$ \\
Depth & $0.0002^{\mathrm{a}}$ & $0.0159^{\mathrm{a}}$ \\
Treatment $\times$ Depth & $0.3537^{\mathrm{a}}$ & $0.6785^{\mathrm{a}}$ \\
Time & $<0.0001^{\mathrm{b}}$ & $0.0003^{\mathrm{a}}$ \\
Treatment $\times$ Time & $0.6217^{\mathrm{a}}$ & $0.2882^{\mathrm{a}}$ \\
Depth $\times$ Time & $<0.0001^{\mathrm{b}}$ & $0.0944^{\mathrm{a}}$ \\
Treatment $\times$ Depth $\times$ Time & $0.4795^{\mathrm{a}}$ & $0.1802^{\mathrm{a}}$ \\
\hline
\end{tabular}

Note: ${ }^{\mathrm{a}}$ Denotes statistically insignificant $(P>0.01) ;{ }^{\mathrm{b}}$ denotes statistically significant $(P \leqslant 0.01)$.

that there was no statistically significant difference in the movement of hormones between the control and the biochar treatment at different depths and times during the second year after biochar application.

\subsection{Fate and transport of estrone}

The mass balance (Table 6) was calculated based on the 
Table 6 Amount of estrone E1 $(\mu \mathrm{g})$ in different soil profile depth ranges and in leachate, and overall, over $46 \mathrm{~d}$ period

\begin{tabular}{|c|c|c|c|c|c|c|c|c|c|c|c|c|}
\hline \multirow{3}{*}{ Day } & \multicolumn{12}{|c|}{ Treatment } \\
\hline & \multicolumn{6}{|c|}{ Soil + Poultry manure (Control) } & \multicolumn{6}{|c|}{ Soil + Poultry manure + Biochar } \\
\hline & $5-10 \mathrm{~cm}$ & $10-20 \mathrm{~cm}$ & $20-50 \mathrm{~cm}$ & $50-70 \mathrm{~cm}$ & Leachate & Total & $5-10 \mathrm{~cm}$ & $10-20 \mathrm{~cm}$ & $20-50 \mathrm{~cm}$ & $50-70 \mathrm{~cm}$ & Leachate & Total \\
\hline 0 & 0.32 & 29.34 & 0.00 & 0.00 & 0.000 & 29.65 & 0.39 & 15.84 & 4.35 & 21.53 & 0.000 & 42.12 \\
\hline 1 & 12.51 & & & & & & 12.46 & & & & & \\
\hline 2 & 6.66 & 2.72 & 4.51 & 0.00 & 0.105 & 13.98 & 5.87 & 9.57 & 0.00 & 0.00 & 0.110 & 15.56 \\
\hline 16 & 4.11 & 3.47 & 5.84 & 6.69 & 0.689 & 20.79 & 2.91 & 4.46 & 12.10 & 6.79 & 0.623 & 26.89 \\
\hline 31 & 5.34 & 17.37 & 11.25 & 10.31 & 0.275 & 44.54 & 3.68 & 16.62 & 2.74 & 27.57 & 0.264 & 50.87 \\
\hline 46 & 1.56 & 1.58 & 3.78 & 5.37 & 0.244 & 12.53 & 2.41 & 0.99 & 3.55 & 4.59 & 0.254 & 11.79 \\
\hline
\end{tabular}

equation of ElSayed et al. ${ }^{[14]}$ given above. The control lost E1 hormone down the soil profile at a faster rate than in the biochar treatment. There were no regular trends in mass change in E1 observed in the leachate. Like the E2 hormone, there was a sudden decrease in the mass of E1 after the first irrigation in both treatments, but the rate of decline in E1 decreased with time. In Fig. 2b, an increase in mass of E1 between Day 16 (second irrigation event) and Day 31 (third irrigation event) is evident. This was due to a biotransformation of $\mathrm{E} 2$ into $\mathrm{E}^{[16]}$. This change was observed more at lower depths, which experienced anaerobic conditions favorable for this biotransformation $^{[23]}$. This change was clear from the decreased mass of E2 between Days 16 and 31 ( Table 4).

During the first year of application, the biochar treatment retained more mass of E1 compared to the control but during the second year after application, the biochar treatment held less mass of E1 (Fig. 2b). Biochar provides a microhabitat for the growth of microbes ${ }^{[24]}$ so there is a higher rate of biotic degradation of E1 in the biochar treatment compared to control (Fig. 3b). Decreases in E1 degradation during the third irrigation event (Fig. 3b) were due to a gain in E1 mass from the biotransformation of E2, which is similar to the findings of Yu et al. ${ }^{[23]}$.

\subsubsection{Concentration of estrone in soil at different depths and times}

Figure 5 shows the concentration of E1 $\left(\mu \mathrm{g} \cdot \mathrm{kg}^{-1}\right)$ in both treatments at different depths and times. The concentration of E1 on Day 1 was high when the poultry manure was applied but then decreased with time.

At all depths, there was some background concentration of E1 from the manure applied during the first year of study when the biochar was freshly applied. There was some increase in concentration of E1 between Days 16 and 31 because of biotransformation of E2 into E1, which was also observed by Xuan et al. ${ }^{[16]}$. This was clear from the decline of E2 concentration (Fig. 4) between these days. The biochar treatment had a higher concentration at these times, which might indicate higher mobility of the hormones. The reason for higher mobility is that the release of dissolved organic carbon from biochar upon physical degradation acted as a carrier of hormones down the soil profile. An increasing number of observations suggests that biochar can be degraded, by both biotic and abiotic processes leading to initial physical fragmentation as discussed above. However, these trends were not evident at lower depths and were difficult to predict as those depths were losing and gaining mass of E1 simultaneously. There were no statistically significant differences observed between control and biochar treatment at different depths and times (Table 5).

\subsection{Concentration of $17 \beta$-estradiol and estrone in leachate}

Concentrations of the hormones collected in the leachate after each irrigation event are shown in Fig. 6. E2 concentration in the control leachate after the first irrigation was greater than E2 concentration in leachate from the biochar treatment (Fig. 6a). This might be because there was a certain amount of E2 left in the lower depths of the soil profile from the poultry application in the previous year, which is also clear from the mass balance (Table 4). During the subsequent irrigation events, the trends seem to be normal with the biochar treatment releasing slightly higher E2 concentrations compared to the control. The concentration of E2 continued to increase in the leachate after each irrigation event, but there was no clear difference in concentration of E1 found in leachate (Fig. 6b; Table 7). Though the concentration of hormones found in leachate was quite low, it is important to note that these hormones, even in trace concentrations, are highly toxic to aquatic animals $^{[25]}$.

\subsection{Scanning Electron Microscopy (SEM)}

Scanning electron microscopy helped analyze the structural differences between fresh biochar and used biochar. From the images shown in Fig. 7, it is clear that the internal and surface pore structure was disturbed in the biochar that had been in the soil for two consecutive years. The temperature at the site fell to $-25^{\circ}$ during winter. Biochar particles can be fragmented into smaller particles when 
(a)

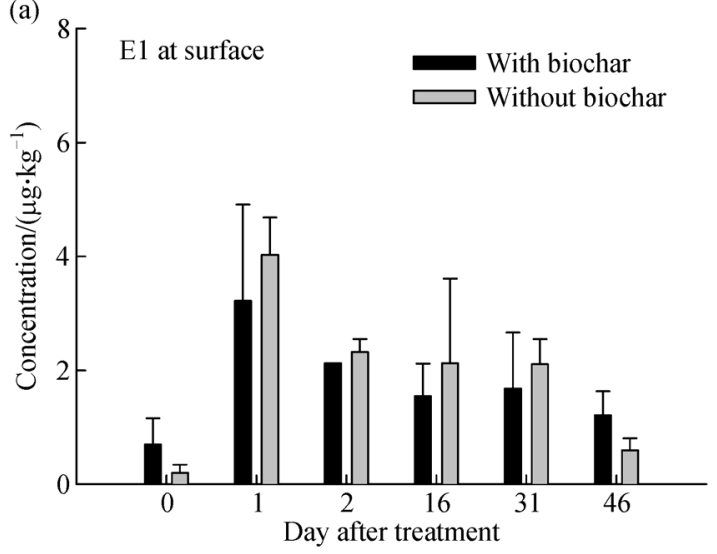

(c)

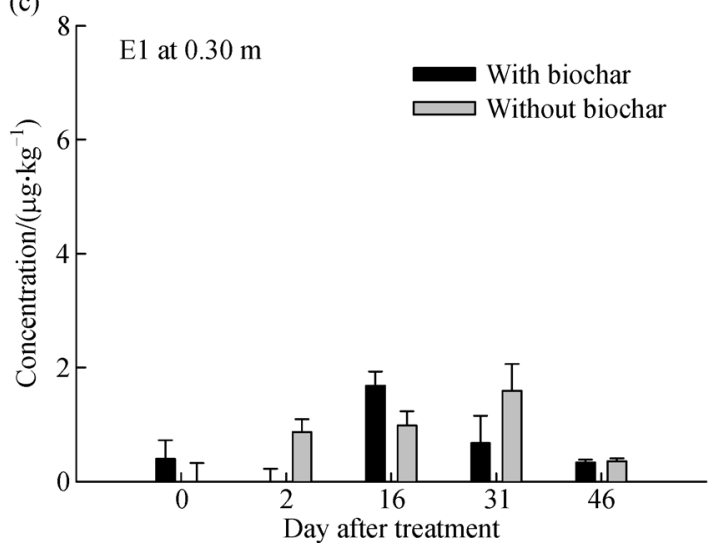

(b)

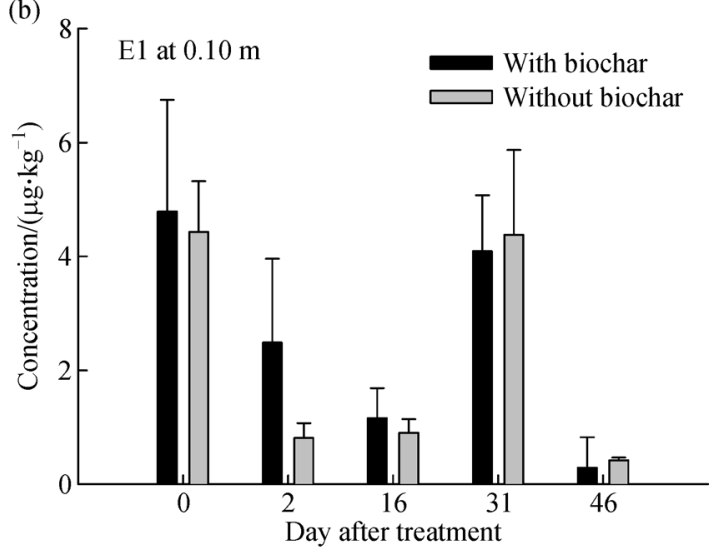

(d)

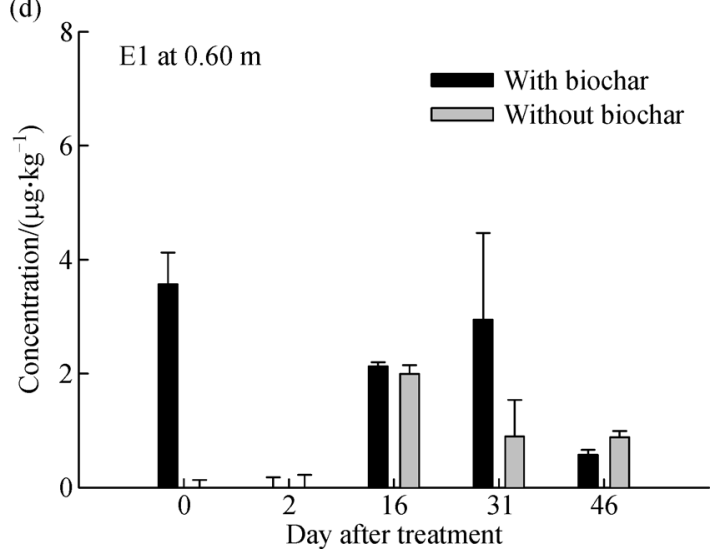

Fig. 5 Estrone E1 $\left(\mu \mathrm{g} \cdot \mathrm{kg}^{-1}\right)$ concentration over a period of 46 days. (a) In the topsoil; (b) at $0.10 \mathrm{~m}$ from surface of soil profile; (c) at $0.30 \mathrm{~m} ;(\mathrm{d})$ at $0.60 \mathrm{~m}$.

(a)

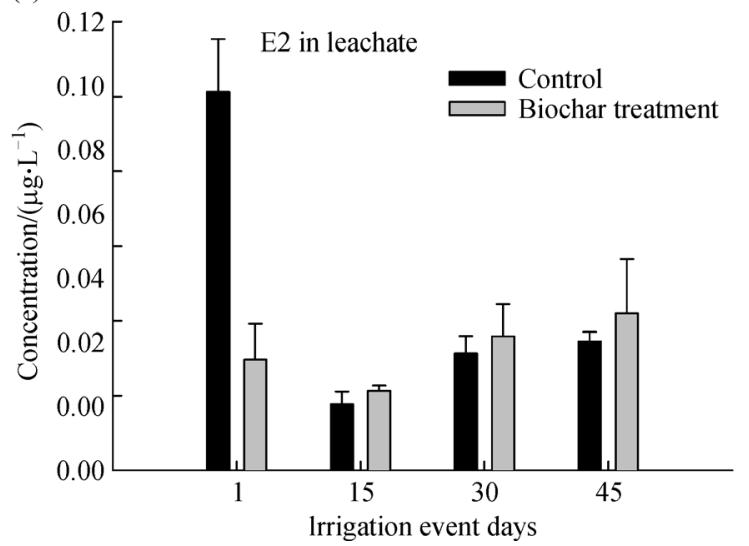

(b)

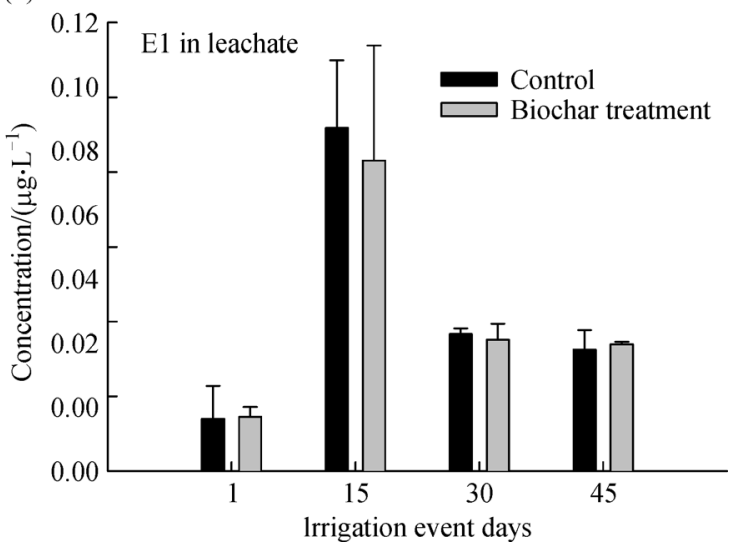

Fig. 6 Concentration in leachate after each of four irrigation events. (a) $17 \beta$-estradiol $(E 2)\left(\mu g \cdot L^{-1}\right)$; (b) estrone $(E 1)\left(\mu g \cdot L^{-1}\right)$. 
Table 7 Analysis of variance for $17 \beta$-estradiol and estrone residue measurements in leachate

\begin{tabular}{lcc}
\hline \multirow{2}{*}{ Effect } & \multicolumn{2}{c}{ Probability } \\
\cline { 2 - 3 } & $17 \beta$-estradiol (E2) & Estrone (E1) \\
\hline Treatment & $0.0719^{\mathrm{a}}$ & $0.6768^{\mathrm{a}}$ \\
Depth & $0.0007^{\mathrm{a}}$ & $<0.0001^{\mathrm{b}}$ \\
Treatment $\times$ Depth & $0.0104^{\mathrm{a}}$ & $0.3442^{\mathrm{a}}$ \\
\hline
\end{tabular}

Note: ${ }^{\mathrm{a}}$ Denotes statistically insignificant $(P>0.01) ;{ }^{\mathrm{b}}$ denotes statistically significant $(P \leqslant 0.01)$.

water penetrates the pores. This causes it to swell during freezing temperatures and forces the biochar particles to break $^{[26]}$. The fragmented biochar also led to the release of dissolved organic carbon ${ }^{[10]}$, which facilitated the mobility of hormones down the soil profile ${ }^{[5]}$.

(a)

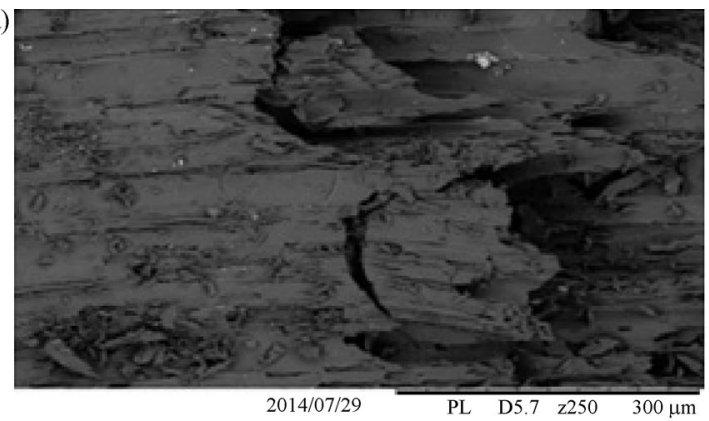

(c)

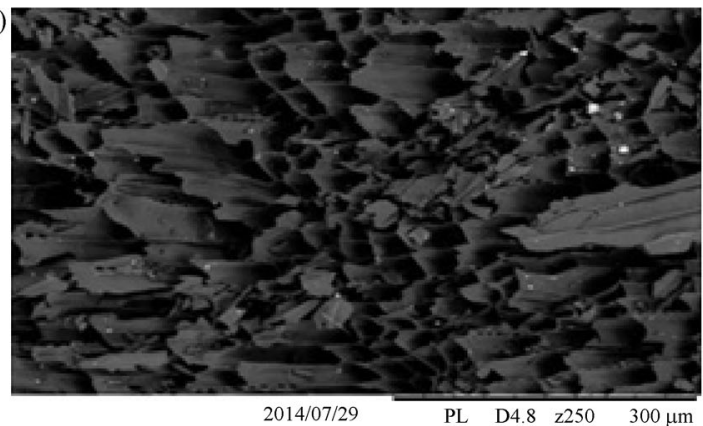

\subsection{Sorption test}

The results for the sorption of pure hormones onto biochar, expressed as the percentage removal of hormones by biochar, are shown in Fig. 8. Less pure hormone was removed from the solution containing used biochar, which means it has a lower capacity to adsorb hormones compared to fresh biochar. This supports the findings presented above that biochar retention capacity for hormones decreases over time.

\section{Conclusions}

Poultry manure is a potential source of estrogen that could have a severe impact on the environment. Biochar has the potential to adsorb these harmful hormones. In this study
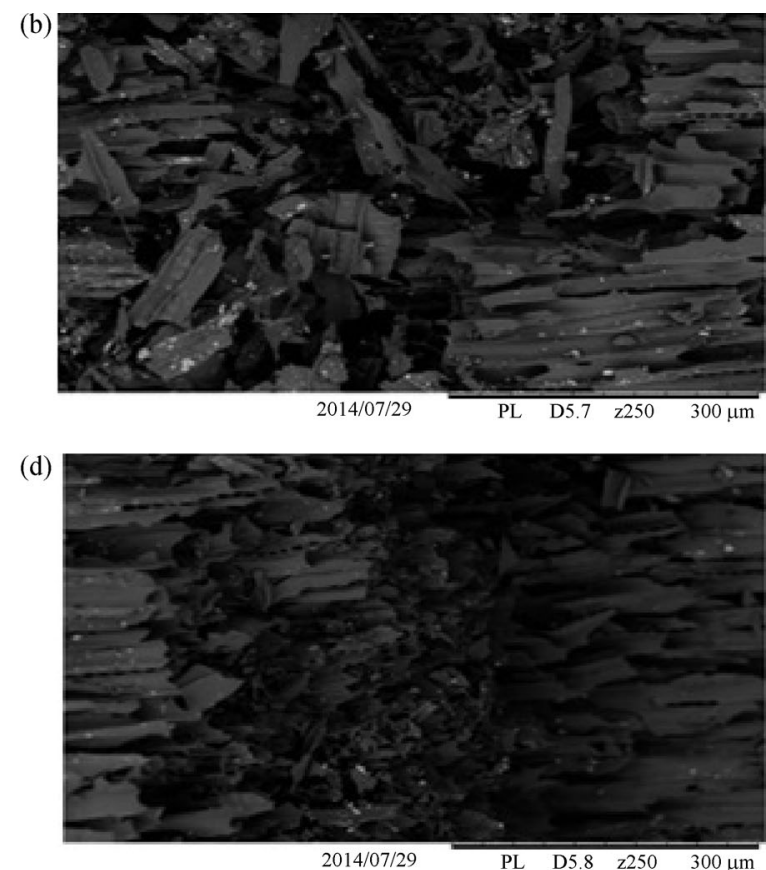

Fig. 7 Scanning electron microscopy (SEM) images of internal surface of fresh biochar (a), internal surface of used biochar (b), outer surface of fresh biochar (c), and outer surface of used biochar (d)
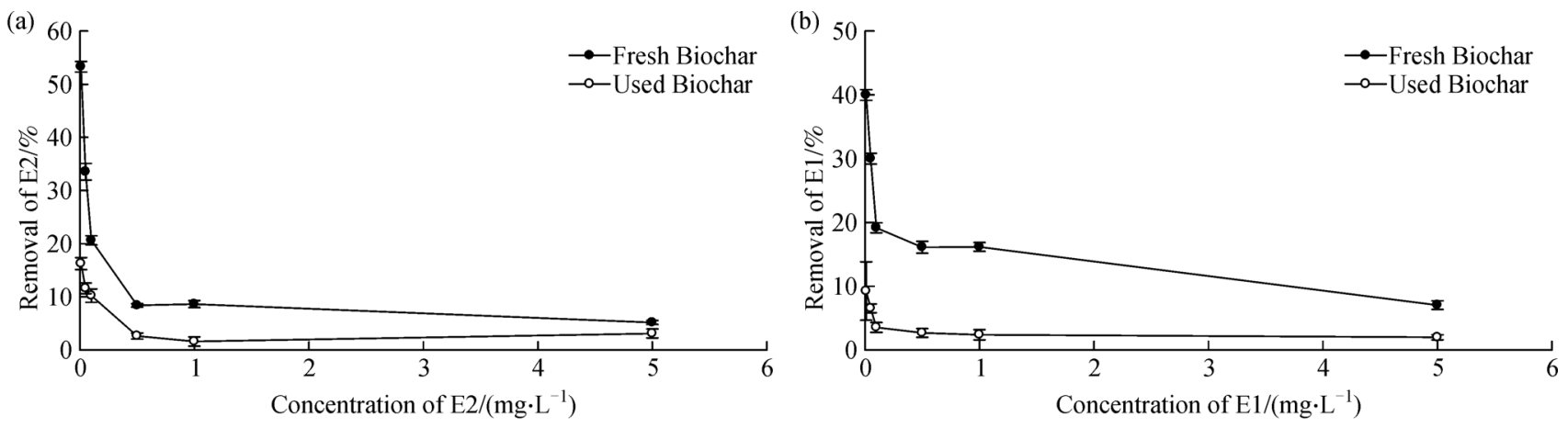

Fig. 8 Percentage removal of hormones by fresh biochar and used biochar. (a) 17ß-estradiol (E2); (b) estrone (E1). 
$1 \%$ slow pyrolysis biochar was tested for adsorption of hormones from poultry manure in sandy soil during the second year of application. During the second year, there was no significant difference observed in movement of hormones in the used biochar treatment and the control. Pore structure on the surface of the biochar underwent various biotic and abiotic changes over time, reducing the hydrophobic interactions of biochar surface with the hormones. Also, the release of dissolved organic carbon further facilitated the mobility of hormones down the soil profile. These findings lead to the conclusion that the retention capacity of $1 \%$ slow pyrolysis biochar for hormones from poultry manure decreases over time. There are gaps in scientific knowledge of biochar properties due to its inconsistent behavior under different field conditions. There is a possibility that the type of biochar used in the current study might perform differently under different conditions. Therefore, there is a need to do further field studies on biochar under a wide range of conditions before it can be widely used for remediation of pollution caused by emerging contaminants.

Acknowledgements The authors would like to acknowledge the financial support provided by the Natural Sciences and Engineering Research Council of Canada (NSERC) under the project Reducing Environmental Pollution from Veterinary Antibiotics, Endocrine Disruptive Chemicals, and Pathogens in Livestock Manure. Also, we would like to thank the Department of Bioresource Engineering, McGill University for providing a Liliane and David Stewart Fellowship in Water Resources for support of this research work.

Compliance with ethics guidelines Sukhjot Mann, Zhiming Qi, Shiv O. Prasher, Lanhai Li, Dongwei Gui, and Qianjing Jiang declare that they have no conflict of interest or financial conflicts to disclose.

This article does not contain any studies with human or animal subjects performed by any of the authors.

\section{References}

1. Choi S M, Yoo S D, Lee B M. Toxicological characteristics of endocrine-disrupting chemicals: developmental toxicity, carcinogenicity, and mutagenicity. Journal of Toxicology and Environmental Health, Part B: Critical Reviews, 2004, 7(1): 1-23

2. Arcand-Hoy L D, Benson W H. Fish reproduction: an ecologically relevant indicator of endocrine disruption. Environmental Toxicology and Chemistry, 1998, 17(1): 49-57

3. Goeppert N, Dror I, Berkowitz B. Detection, fate and transport of estrogen family hormones in soil. Chemosphere, 2014, 95: 336-345

4. Jobling S, Nolan M, Tyler C R, Brighty G, Sumpter J P. Widespread sexual disruption in wild fish. Environmental Science \& Technology, 1998, 32(17): 2498-2506

5. Sarmah A K, Northcott G L, Scherr F F. Retention of estrogenic steroid hormones by selected new zealand soils. Environment International, 2008, 34(6): 749-755

6. Bailey S E, Olin T J, Bricka R M, Adrian D D. A review of potentially low-cost sorbents for heavy metals. Water Research, 1999, 33(11): 2469-2479
7. Ying G G, Kookana R S. Sorption and degradation of estrogen-likeendocrine disrupting chemicals in soil. Environmental Toxicology and Chemistry, 2005, 24(10): 2640-2645

8. Sarmah A K, Srinivasan P, Smernik R J, Manley-Harris M, Antal M J, Downie A, van Zwieten L. Retention capacity of biocharamended new zealand dairy farm soil for an estrogenic steroid hormone and its primary metabolite. Australian Journal of Soil Research, 2010, 48(7): 648-658

9. Schmidt M W I, Noack A G. Black carbon in soils and sediments: analysis, distribution, implications, and current challenges. Global Biogeochemical Cycles, 2000, 14(3): 777-793

10. Joseph S, Lehmann J. Biochar for environmental management: Science and technology, London, GB: Earthscan, 2009

11. Alizadeh S. Use of biochar for reducing female sex hormonal pollution in agricultural areas. Dissertation for the Master Degree. Montreal: McGill University, 2014

12. Hanselman T A, Graetz D A, Wilkie A C. Manure-borne estrogens as potential environmental contaminants: a review. Environmental Science \& Technology, 2003, 37(24): 5471-5478

13. Beegle D. Estimating manure application rates. Agronomy Facts 55, College of Agricultural Sciences, The Pennsylvania State University, University Park, PA, 1997.

14. ElSayed E M, Prasher S O, Patel R M. Effect of nonionic surfactant Brij 35 on the fate and transport of oxytetracycline antibiotic in soil. Journal of Environmental Management, 2013, 116: 125-134

15. Stafiej A, Pyrzynska K, Regan F. Determination of anti-inflammatory drugs and estrogens in water by HPLC with UV detection. Journal of Separation Science, 2007, 30(7): 985-991

16. Xuan R, Blassengale A A, Wang Q. Degradation of estrogenic hormones in a silt loam soil. Journal of Agricultural and Food Chemistry, 2008, 56(19): 9152-9158

17. Lee L S, Strock T J, Sarmah A K, Rao P S C. Sorption and dissipation of testosterone, estrogens, and their primary transformation products in soils and sediment. Environmental Science \& Technology, 2003, 37(18): 4098-4105

18. Casey F X, Simunek J, Lee J, Larsen G L, Hakk H. Sorption, mobility, and transformation of estrogenic hormones in natural soil. Journal of Environmental Quality, 2005, 34(4): 1372-1379

19. Lai K M, Johnson K L, Scrimshaw M D, Lester J N. Binding of waterborne steroid estrogens to solid phases in river and estuarine systems. Environmental Science \& Technology, 2000, 34(18): 3890-3894

20. Yu Z, Huang W. Competitive sorption between $17 \alpha$-ethinyl estradiol and naphthalene/phenanthrene by sediments. Environmental Science \& Technology, 2005, 39(13): 4878-4885

21. Hale S E, Hanley K, Lehmann J, Zimmerman A R, Cornelissen G. Effects of chemical, biological, and physical aging as well as soil addition on the sorption of pyrene to activated carbon and biochar. Environmental Science \& Technology, 2011, 45(24): 1044510453

22. Herman J S, Mills A L. Biological and hydrogeological interactions affect the persistence of $17 \beta$-estradiol in an agricultural watershed. Geobiology, 2003, 1(2): 141-151

23. Yu C P, Deeb R A, Chu K H. Microbial degradation of steroidal estrogens. Chemosphere, 2013, 91(9): 1225-1235 
24. Wei L, Shutao W, Jin Z, Tong X. Biochar influences the microbial community structure during tomato stalk composting with chicken manure. Bioresource Technology, 2014, 154: 148-154

25. Khanal S K S K, Xie B, Thompson M L, Sung S, Ong S K, van Leeuwen $\mathrm{J}$ H. Fate, transport and biodegradation of natural estrogens in the environment and engineered systems. Environmental Science \& Technology, 2006, 40(21): 6537-6546

26. Carcaillet C. Are holocene wood-charcoal fragments stratified in alpine and subalpine soils? Evidence from the Alps based on AMS 14C dates. Holocene, 2001, 11(2): 231-242 